\section{A post mortem assessment of a 25-year-old man with ascending aortic dissection and a novel $M Y L K$ variant}

\author{
Katelyn Hodge, ${ }^{1}$ Katherine G. \\ Spoonamore, ${ }^{2}$ Christopher B. Griffith, ${ }^{1}$ \\ David D. Weaver, ${ }^{1}$ Patricia B.S. Celestino- \\ Soper, ${ }^{1}$ Ty C. Lynnes, ${ }^{1}$ Hongyu Gao, ${ }^{3,4}$ \\ Yunlong Liu, 1,3,4 Matteo Vatta ${ }^{1,2}$ \\ ${ }^{1}$ Department of Medical and Molecular \\ Genetics, Indiana University School of \\ Medicine; ${ }^{2}$ Krannert Institute of \\ Cardiology, Department of Medicine, \\ Indiana University School of Medicine; \\ ${ }^{3}$ Center for Computational Biology and \\ Bioinformatics, Indiana University School \\ of Medicine; ${ }^{4}$ Bioinformatics Program, \\ Indiana University School of Informatics, \\ Indianapolis, IN, USA
}

\section{Abstract}

We report on the process of post mortem evaluation and genetic testing following the death of a 25-year-old man due to ascending aortic dissection leading to aortic rupture. Following the negative clinical testing of a 12gene thoracic aortic aneurysm and dissection panel, research testing revealed a novel c.5732A $>$ T (p.E1911V) variant in exon 34 of the $M Y L K$ gene (NM_053025). Two likely pathogenic variants in this gene have been reported previously in individuals with familial thoracic aortic aneurysm and dissection. Given the unclear clinical consequence of the variant found in our proband, we have classified this change as a variant of uncertain significance. In addition to discussing the complexity involved in variant interpretation, we recognize the need for additional research for more accurate MYLK interpretation. Finally, we comment on the unique challenges of post mortem genetic testing.

\section{Introduction}

Every year, approximately 30,000 deaths in the United States alone are attributed to thoracic aortic dissection and rupture, ${ }^{1}$ which despite being a common cause of mortality, often remains undiagnosed until dissection or death occurs. ${ }^{2}$ The annual reported incidence of aortic dissection ranges from $2.5 / 100,000^{3}$ to as high as 9.1/100,000 and 16.3/100,000 in women and men, respectively. ${ }^{2}$ The true incidence is difficult to determine due to frequent misdiagnosis at the time of death. ${ }^{1}$ The majority of individuals with thoracic aortic dissection (approximately 93\%) are over the age of 40 , with a mean age of 63 years. ${ }^{3}$ Of the individuals with thoracic aortic aneurysm and dissection (TAAD), some are identified to have an underlying genetic syndrome. Disorders such as Marfan, Loeys-Dietz, Ehlers-Danlos, and Turner syndromes all predispose individuals to medial degeneration of the aorta. ${ }^{2}$ Current data suggest nearly $20 \%$ of individuals with TAAD who are otherwise physically normal and without an identified genetic syndrome have at least one first-degree relative with TAAD. ${ }^{3}$ These $20 \%$ of individuals are designated to have familial thoracic aortic aneurysm and dissection (FTAAD).

Advances during recent decades have provided insights into the genetic etiology of both syndromic forms of TAAD and nonsyndromic FTAAD. Currently, genetic testing identifies a molecular cause in about $20 \%$ of individuals with FTAAD, with the majority of genes identified encoding for smooth muscle cell proteins. ${ }^{4}$ Pathogenic changes in ACTA2 account for approximately $14 \%$ of all FTAAD cases. ${ }^{4}$ However, additional genes have been proposed to cause FTAAD. In 2010, Wang et al. reported two variants in the $M Y L K$ gene as likely pathogenic given the predicted damaging effects and the segregation within the families. ${ }^{5}$ Although transcribed in all tissues, MYLK is mostly expressed in smooth muscle. ${ }^{6} M Y L K$ encodes for the myosin light chain kinase (MLCK) enzyme, which plays a crucial role in smooth muscle cell contraction. ${ }^{6}$ Once bound to a $\mathrm{Ca}^{2+} /$ calmodulin complex, MLCK becomes activated and is able to phosphorylate its target protein, the regulatory light chain of myosin II. $^{6}$ Little is known about the potential role of $M Y L K$ variants in aortopathy. The sentinel report by Wang et al. in 2010 represents the first and only publication involving $M Y L K$ variants in FTAAD subjects. ${ }^{5}$ Currently, nine $M Y L K$ variants are listed in the ClinVar database. ${ }^{7}$ Of these nine variants, six are germline variants, and four of these six are reported in association with TAAD (Table 1). ${ }^{7}$ Of note, the p.Arg1480Ter and the p.Ser1759Pro variants are listed from the article published by Wang et al. ${ }^{5}$

Here we report the case of a 25 -year old man who died unexpectedly due to an ascending aortic dissection and rupture. Genetic counseling and testing were performed following his death. Comprehensive next generation sequencing (NGS) analysis using a pan cardiovascular panel including 246 genes has identified a novel c.5732A>T (p.E1911V) variant in exon 34 of the MYLK gene (NM_053025), which we classified as a variant of uncertain significance. In addition to reporting the post mortem assessment, we describe some of the difficulties and limitations associated with post mortem genetic testing and counseling,
Correspondence: Matteo Vatta, 550 University Blvd, UH AOC 6029, Indianapolis, IN 46202, USA. Tel.: 317.944.1066 - Fax: 317.944.4384.

E-mail: mvatta@iu.edu

Key words: $M Y L K$; familial thoracic aortic aneurysm and dissection; post mortem genetic testing; aortopathy.

Acknowledgements: we would like to thank Dr Irmina Gradus-Pizlo for her contribution to the care of these individuals. We would also like to thank the family for their willingness to partici pate in this research.

Contributions: KH, KGS and MV, conceptualized and wrote paper; KGS, provided clinical counseling to the subjects, directed project progress; $\mathrm{CBG}$ and DDW, reviewed and revised paper, PCS, performed next-generation sequencing; TCL, performed Sanger sequencing; $\mathrm{HG}$ and $\mathrm{YL}$, developed NGS bioinformatics pipeline; completed bioinformatics analysis; MV, performed the analysis of next-generation sequencing and Sanger sequencing; provided review and oversight of the project.

Conflict of interest: no conflicts of interest are declared by the authors. KGS, PCS, TCL and MV are supported by the Indiana University Health, Indiana University School of Medicine Strategic Research Initiative.

Received for publication: 28 April 2015.

Revision received: 26 June 2015

Accepted for publication: 30 June 2015.

This work is licensed under a Creative Commons Attribution NonCommercial 3.0 License (CC BYNC 3.0).

(C) Copyright K. Hodge et al., 2015

Licensee PAGEPress, Italy

Cardiogenetics 2015; 5:5251

doi:10.4081/cardiogenetics.2015.5251

and the complexity of aortopathy genetic evaluation.

\section{Materials and Methods}

\section{Clinical phenotype}

The proband was a 25-year old Caucasian male with a history of morbid obesity and hypertension, initially evaluated at an outside institution. He was $195.6 \mathrm{~cm}$ tall and weighed $155.9 \mathrm{~kg}$. According to maternal report, previous medical history includes suspected subaortic stenosis at age 12; however, echocardiogram imaging at age 19 was normal. He had no further cardiologic evaluations at his local institution until the time of his death. He visited the emergency department of a third hospital four days prior to his death with com- 
plaints of back and abdominal pain, shortness of breath, and diarrhea. A computed tomography scan of the abdomen and pelvis was normal with the exception of an umbilical hernia and an atrophic right kidney. He was discharged from the emergency department with a diagnosis of gastroenteritis. Four days later, he returned to the emergency department given his persistent symptoms. An electrocardiogram indicated severe diffuse ST elevation of all leads except aVR, III, and aVF, and PR depression in lead II. During a cardiology consultation, the patient became short of breath, diaphoretic, cyanotic and pulseless. Resuscitation was attempted for an extended period of time and other than one episode of ventricular fibrillation, he had no cardiac electrical activity. Transthoracic ultrasound at the bedside revealed pericardial effusion. On autopsy, he was found to have a ruptured type A aortic dissection from the aortic valve to the abdominal aorta. Additionally, he had dilatation and mild hypertrophy of the left ventricle, a sign of long-standing hypertension. The pathology report showed aortic wall changes consistent with medial degeneration. The coroner noted that there was no evidence of coarctation of the aorta. No further details regarding the size or changes of the aorta were provided. Toxicology analyses on both blood and urine were negative.

Additional medical history was noncontributory. Although no dysmorphologic examination was obtained, in a photograph of the patient, he did not appear to have physical characteristics consistent with a dysmorphic syndrome.

\section{Family history}

A three-generation pedigree was obtained during the genetic counseling session at an Indiana University Health facility with the proband's mother and father (Figure 1). The proband has a living brother and sister, both of whom had normal screening echocardiograms following their brother's death. The brother was found to have mild left ventricular hypertrophy of $12 \mathrm{~mm}$. He had two ventricular septal defects at birth, which subsequently closed.

The proband's mother reported she had a previous echocardiogram suggestive of mitral valve prolapse with regurgitation; however, follow-up echocardiograms were normal. Most recently, both an electrocardiogram and an echocardiogram were normal. The mother also self-reported additional physical features, including mild scoliosis, arachnodactyly, mild pectus excavatum, and mild myopia. She had no other ocular abnormalities, skin laxity, excessive bruising, hypermobility, or smooth/velvety skin. Of note, no individuals in the family received a formal evaluation at their institution to rule out the presence of a connective tissue disorder, though the index of suspicion was low after cardiologist and genet- ic counselor visits. Very little information is known about the more distant maternal relatives. The maternal family is of Scottish and English ancestry.

By echocardiogram, the proband's father had mild enlargement of the sinuses of Valsalva $(4.26 \mathrm{~cm})$, normal sinutubular junction $(3.5 \mathrm{~cm})$, and normal ascending aorta diameter $(3.95 \mathrm{~cm})$. He also had stage 1 diastolic dysfunction, mild right ventricular enlargement, and was borderline tachycardic. An echocardiogram approximately one year later also showed mild dilation at the sinuses of Valsalva $(4.1 \mathrm{~cm})$ and ascending aorta (3.8 $\mathrm{cm}$ ), a small left ventricular cavity, mild concentric left ventricular hypertrophy, and stage 1 to 2 left ventricular diastolic dysfunction. No z-score measurements were calculated in the reports. He is currently taking metformin for type II diabetes mellitus and a prophylactic statin medication and lisinopril for further vascular complications. Additional medical history includes morbid obesity and obstructive sleep apnea. Paternal family history is significant for a paternal grandfather with an abdominal aortic aneurysm detected at age 75 . He also has a history of heavy tobacco use, uncontrolled hypertension, and a myocardial infarction at 77 years of age. The paternal family is of German, Irish, and Swiss ancestry.

\section{Molecular analysis}

Following the patient's death we were able to obtain a frozen blood specimen collected in two red top tubes containing NaF and EDTA. We were able to successfully extract sufficient DNA for subsequent genetic analysis. Following informed consent by the proband's parents, a 12-gene clinical TAAD panel was performed. The test included sequencing of
ACTA2, CBS, COL3A1, COL5A1, COL5A2, FBN1, FBN2, MYH11, SLC2A10, SMAD3, TGFBR1, and TGFBR2. In addition, research-based molecular analysis was performed on the proband, using custom HaloPlex Target Enrichment System (Agilent Technologies, Santa Clara, CA, USA) to capture the coding sequence and intron/exon boundaries of 246 cardiovascular related genes. Targeted regions found to have corresponding pseudogenes elsewhere in the genome were excluded. The total sequenceable design size obtained was of $2.37 \mathrm{Mbp}$ and the recommended minimum sequencing per sample was of $474 \mathrm{Mbp}$ for 200x sequencing coverage depth. Enrichment was validated and target DNA concentration of 175-625 bp size products was determined using the Agilent 2200 TapeStation, while the library was reassessed with the 2200 TapeStation. Approximately $3 \mathrm{pM}$ of pooled libraries spiked with $5 \%$ of $12.5 \mathrm{pM}$ PhiX library (Illumina, Inc., San Diego, CA, USA) were loaded onto an Illumina MiSeq using a standard reagent kit v2 (300 cycles) and standard flow cell. FASTQ files were obtained from the MiSeq instrument for each run. Adapter sequences and low quality bases (trim from the 3' end) were trimmed from the 150 bp pair-end MiSeq reads using Trim Galore (v0.3.2). The resulting reads were mapped to the human reference genome UCSC build hg19 using BWA version 0.7.5 $\mathrm{a}^{8}$ and SAMtools version 0.1.19. ${ }^{9}$ The aligned reads were further realigned and the quality scores were recalibrated using GATK 2.8-1.10 Variants were called using GATK Unified Genotyper restricted to specified target regions. Variants were annotated using ANNOVAR ${ }^{11}$ and the Human Gene Mutation Database (HGMD Professional 2014.2, BIOBASE, Beverly, MA, http://www.hgmd.org/). ${ }^{12,13}$ A final report of

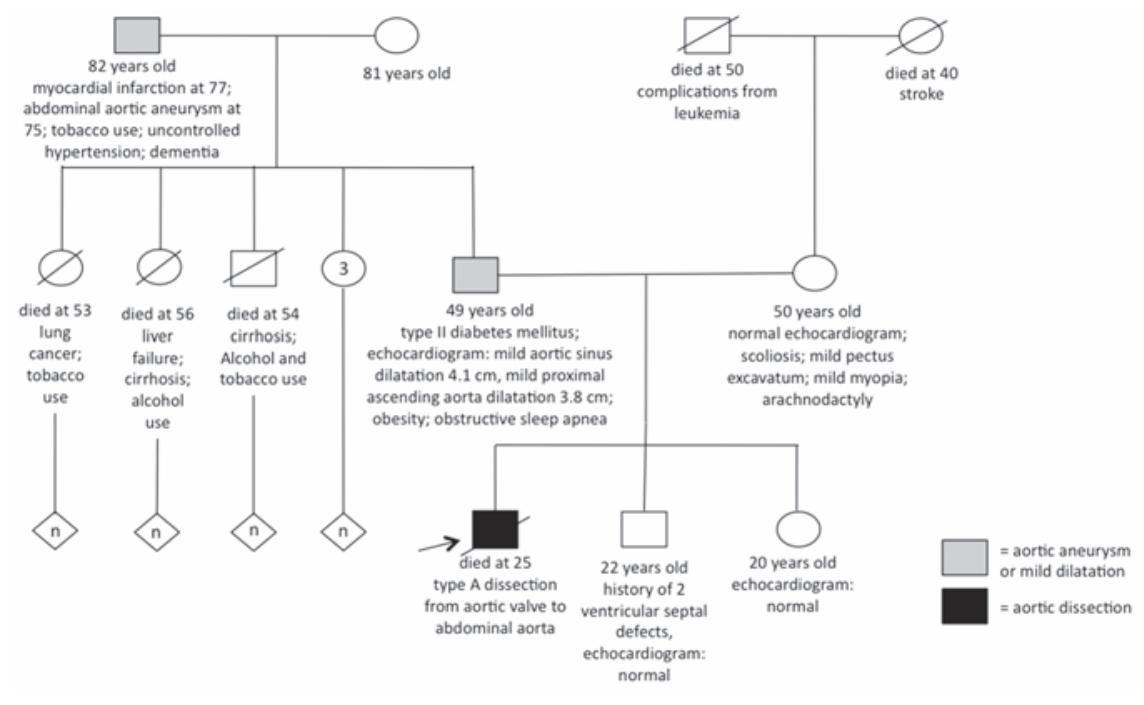

Figure 1. Three-generation pedigree with most recent cardiac imaging. 
variants was generated for the targeted gene regions. The report consists of variant mapping information, gene annotation, amino acid change annotation, variant functional annotation (SIFT, PolyPhen, LRTs, and Mutation Taster), variant evolutionary conservation annotation (PhyloP and GERP++), variant presence and allele frequencies in currently publicly sequenced populations (dbSNP identifiers, 1000 Genomes Project allele, NHLBI-ESP 6500 exome project), and known disease-related functional annotation from HGMD. The various annotation using the aforementioned prediction software provide a cumulative damage score ranging from 0 (no damage) to 6 (highest damage).

\section{Results}

\section{Molecular findings}

Clinical molecular analysis of the 12-gene clinical TAAD panel was negative for any pathogenic variants. The testing did reveal a c.5745G >C (p.E1915D) variant in MYH11, which was reported as likely benign because the amino acid change is conservative, in silico models predict the change not to be damaging, the residue is not conserved across species, and no pathogenic variants have been reported nearby the location of this variant. The research NGS panel confirmed the presence of the MYH11 variant and identified a novel c.5732A > T (p.E1911V) variant in exon 34 of the MYLK gene (NM_053025). Both variants were confirmed by Sanger sequencing. Analyses of the biological parents identified the $M Y L K$ variant was paternally inherited.

\section{Discussion}

Thoracic aortic aneurysm with dissection is a devastating disease with a mortality rate of nearly $97 \%{ }^{4}$ Unfortunately, for many families sudden death is the first sign of disease, ${ }^{2}$ and because of this, it is crucial each case is investigated thoroughly and appropriate genetic assessment is offered in order to determine the likelihood of a hereditary predisposition to developing TAAD. In the previous sections we have described the post mortem assessment of a subject who died following aortic dissection.
However, there are several limitations that should be discussed in depth when pursuing genetic testing and interpreting genetic test results.

The first issue we encountered was the lack of adequate clinical and physical history. All notes and results from clinical tests performed prior to the post mortem evaluation were obtained from a third institution, and we had no available information about a potential history of joint hypermobility or skin hyperextensibility. However, in the case presented here, the autopsy analysis identified a ruptured type A aortic dissection from the aortic valve to the abdominal aorta. Other causes such as toxic etiology or coarctation of the aorta were ruled out by the coroner, warranting a genetic basis to be considered in the differential diagnosis. It is known that dissection can occur even without prior aortic aneurysm. ${ }^{1}$ This was previously reported by Wang et al. in the individuals with $M Y L K$ variants, where aortic dissection occurred with little to no identified enlargement of the aorta. ${ }^{5}$

A second issue to be considered was the availability of adequate tissue specimen. According to the National Association of Medical Examiners, a sample adequate for potential genetic testing should be saved from any individual younger than 40 years of age who dies suddenly and unexpectedly, when there is suspicion of a genetic disease attributing to their death. ${ }^{14}$ Ideally, the sample should be in the form of $5-10 \mathrm{~mL}$ of blood preserved in a $\mathrm{K}_{2}$ EDTA (purple top) tube. ${ }^{14}$ If a sample is saved, next of kin should be informed and proper consent and authorization must be obtained prior to testing. In the present case, our inquiry revealed two red top tubes of frozen blood, which ultimately contained extractable DNA.

A third significant issue was the relatively nonspecific family history. Although the paternal family history appeared suspicious for aortic disease, there were significant confounding environmental factors. The proband's father presented with mild dilatation of the aortic sinuses and ascending aorta; however, his morbid obesity could be a contributing factor, provided that aortic size increases with both age and body surface area. ${ }^{2}$ Similarly, the paternal grandfather was diagnosed with an isolated abdominal aortic aneurysm at age 75, but has a history of heavy tobacco use and uncontrolled hypertension, which are both known risk factors for developing an aortic dissection. ${ }^{2}$ In general, individuals with disease of the ascending aorta do not commonly have family members with disease of the abdominal aorta. ${ }^{1}$ In cases of isolated aortic aneurysm, it is certainly possible that an underlying genetic factor is contributing to the aneurysm in addition to environmental factors. Although our proband's mother reported some physical features consistent with Marfan syndrome such as scoliosis, arachnodactyly, mild pectus excavatum, and myopia, she does not meet clinical diagnostic criteria for Marfan syndrome. ${ }^{15}$ Further, the diagnosis appears unlikely given her negative FBN1 genetic analysis.

Finally, the present case raises questions on proper interpretation of $M Y L K$ variants. In addition to assessing how a change would affect the gene, the complexity in interpretation reflects the fact that little is currently known about this gene and its association with aortopathy. Other than Wang et al., ${ }^{5}$ no other publications have provided additional evidence supporting this association. It is known that MLCK is an integral component of the smooth muscle cell contractile apparatus. ${ }^{6}$ Therefore, it is reasonable to support the idea that deleterious mutations in $M Y L K$ could contribute to aortic wall degeneration in a manner similar to ACTA2 and MYH11

Table 1. Germline variants in $M Y L K$ reported in ClinVar.

\begin{tabular}{lll} 
Variant & Clinical phenotype & Suggested clinical significance \\
p.Gly1360Asp & TAAD & Uncertain significance \\
p.Gly866Ser & TAAD; disproportionate tall stature & Uncertain significance \\
\hline p.Arg1480Ter & TAAD & Pathogenic/likely pathogenic \\
p.Ser1759Pro & TAAD & Pathogenic/likely pathogenic \\
3q13.33-21.2 deletion & Developmental delay & Pathogenic/likely pathogenic \\
3q13.32-21.2 deletion & Developmental delay & Pathogenic/likely pathogenic \\
\hline
\end{tabular}

TAAD, thoracic aortic aneurysm and dissection.

Table 2. Next generation sequencing variant results after Sanger confirmation in genes associated with thoracic aortic aneurysm and dissection.

\begin{tabular}{lccccc} 
Gene & Accession number & Bxon & Nucleotide change & Amino acid change & Damage score (0-6) \\
MYH11 & 002474 & 40 & c.5745G>C & p.Glu1915Asp & 2 \\
MYLK & 053025 & 34 & c.5732A>T & p.Glu1911Val & 2 \\
\hline
\end{tabular}


mutations. ${ }^{5}$ Although we do not have functional data for the p.E1911V variant, the MYLK variants reported by Wang et al. demonstrated an abnormal kinase binding to calmodulin and reduced kinase activity, thus providing a substrate for acute aortic dissection with negligible or no aortic enlargement prior to dissection. ${ }^{5}$ Loss-offunction mutations resulting in decreased MLCK product may result in decreased contractile ability of the smooth muscle cell. ${ }^{5}$ This is in part due to the observed increased proteoglycan deposition in the aortic media, increased type III collagen, and increased expression of the elastindegrading metalloproteinase, which have been consistently reported to be overexpressed in ascending aortic aneurysms. ${ }^{16}$ Because of the need for future investigation on $M Y L K$ and its role in FTAAD, we currently consider this gene to be one of uncertain significance with regard to causing FTAAD. The clinical significance of the $M Y L K$ variant found in the proband and his father is currently unclear. Although it is certainly interesting that the father has this variant and dilatation of the ascending aorta, we cannot, at this time, determine if the two are associated. Similarly, because this variant appears to be paternally inherited in the proband, it is possible the paternal grandfather with an abdominal aneurysm also carried this variant. As noted previously, it is uncommon for an individual with familial disease of the ascending aorta to have a relative with isolated disease of the abdominal aorta, ${ }^{1}$ but we cannot rule out a genetic predisposition that, in addition to environmental factors, was sufficient to cause his aneurysm. Genetic testing of the grandfather was not discussed with the family. The MYLK variant remains in our opinion the most suggestive candidate. The fact that the $M Y L K$ variant was not seen in 2500 subjects of the 1000 Genomes Browser (http//browser.1000genomes.org/index.html), in 6500 subjects of the NHLBI Exome Sequencing Project (ESP) database (http://evs.gs.washington.edu/EVS/), or in 60,689 unrelated individuals of the Exome Aggregation Consortium (ExAC) (Cambridge, MA, http:/exac.broadinstitute.org), which evaluated exome data from individuals of European, African American, Hispanic, Asian and other backgrounds, is suggesting, yet not supporting, its possible pathogenicity. Moreover, although both SIFT and Polyphen2 software predictions were concordant in defining the variant as damaging, our cumulative damage score was low (2/6). Despite the sensitivity of SIFT and PolyPhen being quite high (69\% and 68\%, respectively), their specificity is low ( $13 \%$ and $16 \%$, respectively), and although they appear to be reliable in predicting a deleterious effect in loss-of-function variants, ${ }^{17}$ caution must be exercised due to a possible overestimation of pathogenicity. There are limitations to the genetic testing that was performed on the proband. One limitation to both the clinical and research testing is that deletion/duplication analysis was not performed. We therefore, cannot rule out a deletion or duplication as the cause for the dissection. Of note, four genes have been added to the clinical TAAD panel since the time the proband's testing was performed. One gene that has since been added to the clinical panel that was not included on our 246-gene research panel is the SKI gene.

Variants of uncertain significance (VUS) are often thought to be both difficult to interpret and difficult to provide counseling over. In most situations, families who pursue genetic testing are looking for an answer as to what caused the condition in their family. A family or provider may additionally be looking for an answer to help determine appropriate surveillance moving forward. When the result of the genetic test is a VUS, it may make the determination of future surveillance more complicated. In the case of $\mathrm{TAAD}$, guidelines have been set in place for situations in which a definitive genetic diagnosis is not made. In the case presented here, without an identified cause and without definitively knowing from which side of the family a predisposition may have been inherited, the proband's family was counseled and medical recommendations were provided in accordance to the American College of Cardiology Foundation/American Heart Association Guidelines. ${ }^{3}$ Following the death of the proband, all first-degree relatives underwent cardiac imaging in the form of echocardiography. In this family and other FTAAD families, given the reduced penetrance and variable expressivity present in FTAAD, it is recommended that firstdegree relatives have additional screening approximately every two years. ${ }^{3}$

Despite the limitations of post mortem testing, it is important to raise awareness of the process and its availability following aortic dissection and rupture cases that tragically end in death. The potential answers which may be obtained through genetic analysis of the affected individual may be crucial in aiding assessment of family members' risks. Given that $M Y L K$ is becoming increasingly common on clinical TAAD genetic testing panels, we recognize the need for further exploration of this gene in addition to the information that we have presented.

\section{References}

1. Elefteriades J, Farkas E. Thoracic aortic aneurysm: clinically pertinent controversies and uncertainties. J Am Coll Cardiol 2010;55:841-57.

2. Goldfinger J, Halperin J, Marin M, et al.
Thoracic aortic aneurysm and dissection. J Am Coll Cardiol 2014;64:1725-39.

3. Hiratzka L, Bakris G, Beckman J, et al. ACCF/AHA/AATS/ACR/ASA/SCA/SCAI/SIR/STS /SVM guidelines for the diagnosis and management of patients with thoracic aortic disease. J Am Coll Cardiol 2010;55:27-129.

4. Saenen JB, Van Craenenbroeck EM, Proost $\mathrm{D}$, et al. Genetics of sudden cardiac death in the young. Clin Genet 2014;1-13.

5. Wang L, Dong-chuan G, Cao Jiumei, et al. Mutations in myosin light chain kinase cause familial aortic dissections. Am J Hum Genet 2010;87:701-7.

6. Kamm K, Stull J. Dedicated myosin light chain kinases with diverse cellular functions. J Biol Chem 2001;276:4527-30.

7. ClinVar. Available from: http://www. ncbi.nlm.nih.gov/clinvar/, 2014.

8. Li H, Durbin R. Fast and accurate short read alignment with Burrows-Wheeler transform. Bioinformatics 2009;25:1754-60.

9. Li H, Handsaker B, Wysoker A, et al. The Sequence Alignment/MAP format and SAMtools. Bioinformatics 2009:25;2078-9.

10. McKenna A, Hanna M, Banks E, et al. The Genome Analysis Toolkit: A MapReduce framework for analyzing next-generation DNA sequencing data. Genome Res 2010;20:1297-1303.

11. Wang K, Li M, Hakonarson H. ANNOVAR: functional annotation of genetic variants from high-throughput sequencing data. Nucleic Acids Res 2010;38:e164.

12. Stenson PD, Mort M, Ball EV, et al. The Human Gene Mutation Database: 2008 update. Genome Med 2009;1:13.1-13.6.

13. Stenson PD, Mort M, Ball EV, et al. The Human Gene Mutation Database: building a comprehensive mutation repository for clinical and molecular genetics, diagnostic testing and personalized genomic medicine. Hum Genet 2014;133:1-9.

14. Middleton O, Baxter S, Demo E, et al. National association of medical examiners position paper: retaining post mortem samples for genetic testing. Acad Forensic Pathol 2013;3:191-4.

15. Loeys B, Dietz H, Braverman A, et al. The revised Ghent nosology for the Marfan syndrome. J Med Genet 2010;47:476-85.

16. Lesauskaite V, Tanganelli P, Sassi C, et al. Smooth muscle cells of the media in the dilatative pathology of ascending thoracic aorta: morphology, immunoreactivity for osteopontin, matrix metalloproteinases, and their inhibitors. Hum Pathol 2001;32:1003-11.

17. Flanagan SE, Patch AM, Ellard S. Using SIFT and PolyPhen to predict loss-of-function and gain-of-function mutations. Genet Test Mol Biomarkers 2010;14:533-7. 